\title{
Structure properties of pyrolytic lignin extracted from aged bio-oil
}

\author{
JIANG XiaoXiang $^{1}$, NAOKO Ellis ${ }^{2} \&$ ZHONG ZhaoPing ${ }^{1 *}$ \\ ${ }^{1}$ Thermoenergy Engineering Research Institute, Southeast University, Nanjing 210096, China; \\ ${ }^{2}$ Department of Chemical and Biological Engineering, University of British Columbia, Vancouver V6T 1Z3, Canada
}

Received April 21, 2010; accepted January 10, 2011

\begin{abstract}
Fast pyrolysis is a promising technology that can convert biomass into liquid. Bio-oil is one such product, known not only as a greenhouse gas-neutral energy source, but also an opportunity to reduce reliance on fossil fuels. Pyrolytic lignin, a fine homogeneous powder, is the water-insoluble fraction of bio-oil and it contributes to the instability of bio-oil. Additionally, pyrolytic lignin can be used in commercial materials such as adhesives in the wood-based panel industry. This paper presents the structural characterization of pyrolytic lignin extracted from aged bio-oil and the relationship between its properties and the treatment temperature of the aged bio-oil. Pyrolytic lignin samples were characterized by Fourier transform infrared spectroscopy, gel permeation chromatography, differential scanning calorimetry, thermogravimetric analysis and proton nuclear magnetic resonance spectroscopy. The average molecular weight of pyrolytic lignin increased from 700 to $1000 \mathrm{~g} / \mathrm{mol}$ with increasing aging temperature $\left(6-50^{\circ} \mathrm{C}\right)$. Differential scanning calorimetry showed that the glass transition temperature of pyrolytic lignin increases with lower heating rate and higher treatment temperature of bio-oil. An increase in the initial decomposition temperature and the temperature at $95 \mathrm{wt} \%$ weight loss of the aged pyrolytic lignin in thermogravimetry were observed for the bio-oil aged at higher temperature. An increase in residue weight of aged pyrolytic lignin was found in bio-oil aged at higher temperatures.
\end{abstract}

pyrolytic lignin, instability, bio-oil, aging, DSC, FTIR, TGA

Citation: Jiang X X, Naoko E, Zhong Z P. Structure properties of pyrolytic lignin extracted from aged bio-oil. Chinese Sci Bull, 2011, 56: 1417-1421, doi: $10.1007 / \mathrm{s} 11434-011-4465-4$

Due to concerns regarding environmental strain caused by the use of fossil fuels and global warming stemming from the carbon dioxide released from petroleum consumption, renewable sources of energy and chemical feed stocks are attracting increasing attention. Bio-oil obtained by the pyrolysis of biomass is thus emerging as an alternative source of sustainable energy for diesel engines, gas turbines, heating applications and for use as a chemical feed stock [1-3]. The benefit of bio-oil lies not only in its use as a greenhouse gas (GHG)-neutral energy source but also in the opportunity it presents to reduce reliance on fossil fuels.

Bio-oil is known to be unstable due to its volatility and high oxygen content [4], reflected in the increase in viscosity and phase separation between water-rich and organicrich phases over time. The increase in viscosity is shown to

*Corresponding author (email: zzhong@ seu.edu.cn) correlate with increasing average molecular weight [5]. Bridgwater [1] showed that the amount of low molecular weight compounds decreased, while the amount of higher molecular weight compounds increased upon aging. It has been reported that upon aging of bio-oil, pyrolytic lignin contributes to the instability of bio-oil [6,7]. Compared with the native lignin of wood, pyrolytic lignin consists of relatively low molecular weight components due to fragmentation during pyrolysis [8]. It may be dissolved in bio-oil by the water-soluble molecules forming aggregates [9]. With excess amounts of water, pyrolytic lignin precipitates out of solution. Ba et al. [9] have characterized the water insoluble fraction of bio-oil (WIF) using thermo-gra- vimetric analysis (TGA) and differential scanning calorimetry (DSC). The effect of aging on the high-molecular- mass fraction of the WIF is shown through polymerization and condensation reactions of carbohydrate constituents, aldehydes and 
ketones. Changes in water content, viscosity, molecular weight, and infrared spectra have been reported for whole bio-oil from oak feed after storage at 37,60 and $90^{\circ} \mathrm{C}$ for $84 \mathrm{~d}, 9 \mathrm{~d}$ and $15 \mathrm{~h}$, respectively [10]. Aging of bio-oil increased the absorbance in Fourier transform infrared spectroscopy (FTIR) corresponding to hydroxyl, carbonyl, $\mathrm{C}-\mathrm{O}-\mathrm{C}$ and $\mathrm{C}-\mathrm{O}-\mathrm{H}$ groups, suggesting the occurrence of condensation reactions through etherification or esterification during storage.

Information from [6,7] indicates that pyrolytic lignin plays a crucial role in the aging process. Despite its significant effect on the overall physicochemical characteristics of bio-oil over time, detailed changes in the characteristics of pyrolytic lignin during the aging of bio-oil have not been reported. Investigation of the mechanism of aging related to functionality may provide important information for its potential use. This paper reports on the aging of bio-oil at three temperatures: 6,35 and $50^{\circ} \mathrm{C}$. Pyrolytic lignin are then extracted and characterized by FTIR, gel permeation chromatography (GPC), TGA, DSC and proton nuclear magnetic resonance spectroscopy $\left({ }^{1} \mathrm{H}\right.$ NMR).

\section{Experimental}

\subsection{Bio-oil storage and aging test}

Bio-oil sample was supplied by the VTT Company, Finland. The feed stock was pine. The bio-oil was separated into 500 $\mathrm{mL}$ air-tight jars, blanketed with $\mathrm{N}_{2}$ and kept in dark storage at $6^{\circ} \mathrm{C}$. The bio-oil was aged by placing the sample containers in 35 and $50^{\circ} \mathrm{C}$ oil baths, respectively, for $60 \mathrm{~d}$.

\subsection{Pyrolytic lignin extraction}

Pyrolytic lignin was extracted according to the method described in $[7,11,12]$. Five grams of aged bio-oil was added dropwise to $500 \mathrm{~mL}$ of ice-cooled distilled water under stirring at $5000 \mathrm{r} / \mathrm{min}$ with a homogenizer. After stirring for $1 \mathrm{~h}$, the precipitated lignin was filtered and washed. The filtered lignin was re-suspended in $500 \mathrm{~mL}$ of ice-cooled water and stirred for $4 \mathrm{~h}$. Finally, the solution was filtered and the pyrolytic lignin was dried under vacuum at room temperature.

\subsection{Pyrolytic lignin characterization methods}

Elemental analysis was performed using a Perkin Elmer 2400 Series II CHNS/O analyzer (CHN mode) in accordance with the standard procedure. The higher heating values were calculated by Dulong's formula:

$$
\begin{aligned}
& \operatorname{HHV}(\mathrm{MJ} / \mathrm{kg})= \\
& {[338.2 \times \% \mathrm{C}+1442.8 \times(\% \mathrm{H}-\% \mathrm{O} / 8)] \times 0.001 .}
\end{aligned}
$$

FTIR was performed on a Perkin Elmer Spectrum One
FTIR spectrophotometer. Two micrograms of pyrolytic lignin was ground with $150 \mathrm{mg}$ of $\mathrm{KBr}$ and the resulting mixture was pressed into a pellet. All spectra were normalized to the height of the aromatic skeletal vibrations peak at 1432 $\mathrm{cm}^{-1}$. For each sample, 32 spectra were accumulated between 4000 and $400 \mathrm{~cm}^{-1}$.

Molecular weight was estimated by GPC using an Agilent 1100 HPLC system equipped with an autosampler, an isocratic pump, a thermostatically controlled column compartment, a multiple wavelength UV detector and two Waters styragel columns (HR5E and HR1) in tandem. $1 \mathrm{mg}$ of pyrolytic lignin sample was dissolved in $1 \mathrm{~mL}$ of THF, and $50 \mu \mathrm{L}$ of the solution was injected. The THF flow rate was $1 \mathrm{~mL} / \mathrm{min}$ and the temperature of the columns, calibrated with polystyrene standards, was $50^{\circ} \mathrm{C}$. The pyrolytic lignin samples and polystyrene standards were detected at 280 and $254 \mathrm{~nm}$, respectively.

DSC was performed on a TA instruments Q 1000 DSC under nitrogen with a flow rate of $50 \mathrm{~mL} / \mathrm{min}$. Approximately $3 \mathrm{mg}$ of sample was heated from 40 to $250^{\circ} \mathrm{C}$ and cooled to $0^{\circ} \mathrm{C}$ at a rate of $20^{\circ} \mathrm{C} / \mathrm{min}$ to eliminate its strain history. Afterwards, the sample was heated again from 0 to $250^{\circ} \mathrm{C}$ at a rate of $20^{\circ} \mathrm{C} / \mathrm{min}$ for data collection. The glass transition temperature $\left(T_{\mathrm{g}}\right)$ was recorded as the midpoint temperature of the heat capacity transition of the second heating run.

TGA was performed using a TA instruments Q500 TGA. About $3 \mathrm{mg}$ of fine pyrolytic lignin was heated from 40 to $600^{\circ} \mathrm{C}$ at a rate of $10^{\circ} \mathrm{C} / \mathrm{min}$ under a nitrogen flow rate of $50 \mathrm{~mL} / \mathrm{min}$.

For each sample, $200 \mathrm{mg}$ of pyrolytic lignin was suspended in $5 \mathrm{~mL}$ of pyridine. Five milliliters of acetic anhydride were added and the resulting solution was stirred for 48 hours. The acetylated pyrolytic lignin was filtered, poured over ice and washed with cold water to remove the pyridine/acetic anhydride solution. The wet pyrolytic lignin was dried at $22^{\circ} \mathrm{C}$ under vacuum for $48 \mathrm{~h}$. A 100 -mg sample of dried pyrolytic lignin was dissolved in $350 \mu \mathrm{L}$ of deuterated chloroform for a standard ${ }^{1} \mathrm{H}-\mathrm{NMR}$ experiment on a Bruker Avance 300 spectrometer.

\section{Results}

The pyrolytic lignin samples extracted from aged bio-oil at 6,35 and $50^{\circ} \mathrm{C}$ were identified as lignin 06 , lignin 35 and lignin 50, respectively. After extraction, the samples were dried at $25^{\circ} \mathrm{C}$ to avoid side reactions which may occur if heated to higher temperature. The yields of pyrolytic lignin are given in Table 1 . There was an increase in the yield from $14.2 \%$ to $19.1 \mathrm{wt} \%$ with the increasing storage temperature of treated bio-oil. While the oxygen content increased with aging temperature, which implies that oxidation occurred during aging. The hydrogen content showed no significant change. Figure 1 shows the FTIR spectra of the pyrolytic lignin from the aged bio-oils. Peaks of interest are listed in Table 2. 
Table 1 Yield and elemental analysis data of pyrolytic lignin and spruce milled wood lignin (MWL)

\begin{tabular}{|c|c|c|c|c|c|c|c|}
\hline \multirow{2}{*}{ Sample } & \multirow{2}{*}{ Yield (wt\%) } & \multicolumn{2}{|c|}{$\mathrm{C}$} & \multicolumn{2}{|c|}{$\mathrm{H}$} & \multicolumn{2}{|c|}{$\mathrm{O}^{\mathrm{a})}$} \\
\hline & & $\mathrm{wt} \%$ & $\mathrm{C} / \mathrm{C} 06$ & $\mathrm{wt} \%$ & H/H06 & $\mathrm{wt} \%$ & $\mathrm{O} / \mathrm{O} 06$ \\
\hline Lignin 06 & $14.2 \pm 1.4$ & $69.9 \pm 1.2$ & 1.00 & $6.1 \pm 0.2$ & 1.00 & $24.0 \pm 1.4$ & 1.00 \\
\hline Lignin 35 & $17.4 \pm 0.6$ & $68.5 \pm 0.4$ & 0.98 & $6.1 \pm 0.1$ & 1.00 & $25.4 \pm 0.4$ & 1.06 \\
\hline Lignin 50 & $19.1 \pm 0.4$ & $65.9 \pm 0.2$ & 0.94 & $5.8 \pm 0.1$ & 0.95 & $28.3 \pm 0.3$ & 1.18 \\
\hline MWL (spruce) & & 59.98 & & 5.79 & & 33.89 & \\
\hline
\end{tabular}

a) Oxygen by difference.

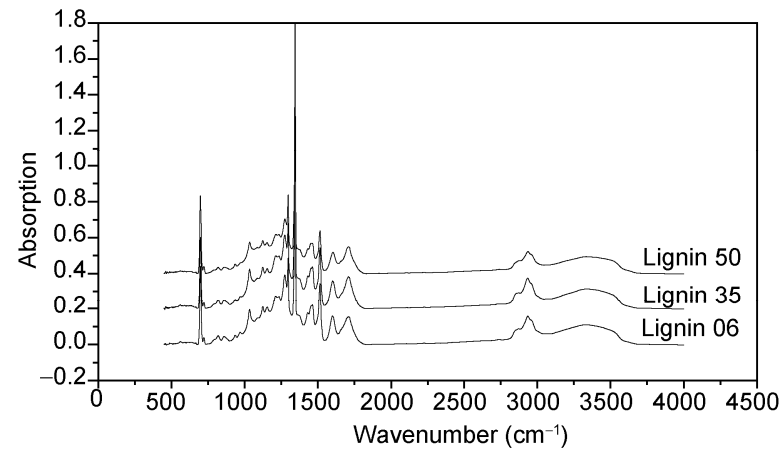

Figure 1 FTIR of pyrolytic lignin from aged bio-oil.

Table 2 FTIR peaks of interest

\begin{tabular}{cccc}
\hline \multirow{2}{*}{ Band origin } & \multicolumn{3}{c}{ Wave-numbers of samples $\left(\mathrm{cm}^{-1}\right)$} \\
\cline { 2 - 4 } & Lignin 06 & Lignin 35 & Lignin 50 \\
\hline O-H stretch & 3334 & 3335 & 3335 \\
C-H stretch & 2935 & 2934 & 2936 \\
C=O stretch & 1710 & 1709 & 1710 \\
Ring stretches & 1601 & 1602 & 1603 \\
Ring stretches & 1514 & 1514 & 1515 \\
$\mathrm{CH}_{3}$, CH ${ }_{2}$ deformations & 1463 & 1463 & 1463 \\
Ring stretch & 1431 & 1432 & 1432 \\
$\mathrm{CC}_{\text {bridge bond stretch }}$ & 1297 & 1297 & 1297 \\
$\mathrm{C}=$ O+guaiacyl ring & 1275 & 1274 & 1275 \\
C-C plus C-O & 1235 & 1234 & 1234 \\
Aromatic C-H defortion & 1154 & 1154 & 1154 \\
in guaiacyl ring & & & 1124 \\
Aromatic C-H defortion & 1124 & 1124 & \\
in syringyl ring & & &
\end{tabular}

The FTIR spectra show characteristic vibrations of typical lignocellulosic materials groups $\mathrm{O}-\mathrm{H}\left(3000-3600 \mathrm{~cm}^{-1}\right)$, $\mathrm{C}-\mathrm{H}\left(2850-3100 \mathrm{~cm}^{-1}\right)$ and $\mathrm{C}=\mathrm{O}\left(1650-1900 \mathrm{~cm}^{-1}\right)$. The bands observed at $600-1030 \mathrm{~cm}^{-1}$ are attributed to hemicelluloses and silicates. In general, the intensity of various bands increased with the increasing aging temperature of bio-oil. Comparison of the $\mathrm{O}-\mathrm{H}$ stretching region (3000-3600 $\mathrm{cm}^{-1}$ ) indicates that a lower temperature increases the $\mathrm{O}-\mathrm{H}$ content. This may be due to $\mathrm{H}$-atom transfer and phenol formation from the phenoxy radical produced from the initial $\beta-\mathrm{O}-4$ ether bond homolysis. At all three aging temperatures, the maximum intensity of the carbonyl region occurs at about the same wavenumbers.
These bands correspond to the formation of unconjugated carbonyls $\left(1710 \mathrm{~cm}^{-1}\right)$, esters $\left(1735 \mathrm{~cm}^{-1}\right)$, and aromatic esters $\left(1770 \mathrm{~cm}^{-1}\right)$, respectively. The increasing $\mathrm{C}-\mathrm{O}$ and carbonyl $\mathrm{C}=\mathrm{O}$ peaks indicate that oxidation occurs at higher storage temperatures, which is supported by the elemental analyses and percentage yields (Table 1).

The structure of pyrolytic lignin was also investigated using GPC, by which the molecular weight distribution (MWD) can also be obtained. Unlike milled wood lignin and most other technical lignin, because of its total solubility in the GPC solvent tetrahydrofuran (THF), pyrolytic lignin did not need to be acetylated for GPC analysis. The retention time determined by GPC depends not only on the molecular size of the molecule but also on the functional groups present. As a result, it is crucial that standards, which possess the same functional groups as the compounds to be analyzed, are used to obtain calibration curves for GPC measurements. Unfortunately, because pyrolytic lignin consists of a complex mixture of various compounds containing a large number of different functional groups, suitable standards for GPC of pyrolytic lignin are not readily available. Furthermore, the absolute value of the molecular weight determined by GPC for the lignin may possess a certain systematic error. Thus, the average molecular which contains the same systematic error, has been compared. Various molecular weight numbers were calculated from the experimental data. The weight average $\left(M_{w}\right)$ and the number average $\left(M_{n}\right)$ molecular weight of the pyrolytic lignin are shown in Table 3, along with the polydispersity ( $P D$ $\left.=M_{w} / M_{n}\right)$.

Figure 2 shows DSC scans of the pyrolytic lignin from the aged bio-oils treated at different temperatures. Thermal analysis was used to analyze the effect of bio-oil storage temperature on the $T_{\mathrm{g}}$ of the pyrolytic lignin. As expected, a higher storage treatment temperature increased the $T_{\mathrm{g}}$ of the pyrolytic lignin. The rising $T_{\mathrm{g}}$ is most likely due to the incorporation of the polar carbonyl groups. The $T_{\mathrm{g}}$ is inversely proportional to the free volume because polar groups

Table 3 Molecular weight distribution of pyrolytic lignin

\begin{tabular}{cccc}
\hline Samples & $M_{n}(\mathrm{~g} / \mathrm{mol})$ & $M_{w}(\mathrm{~g} / \mathrm{mol})$ & $P D\left(M_{w} / M_{n}\right)$ \\
\hline Lignin06 & 710 & 970 & 1.37 \\
Lignin35 & 850 & 1250 & 1.47 \\
Lignin50 & 1010 & 1580 & 1.56 \\
\hline
\end{tabular}




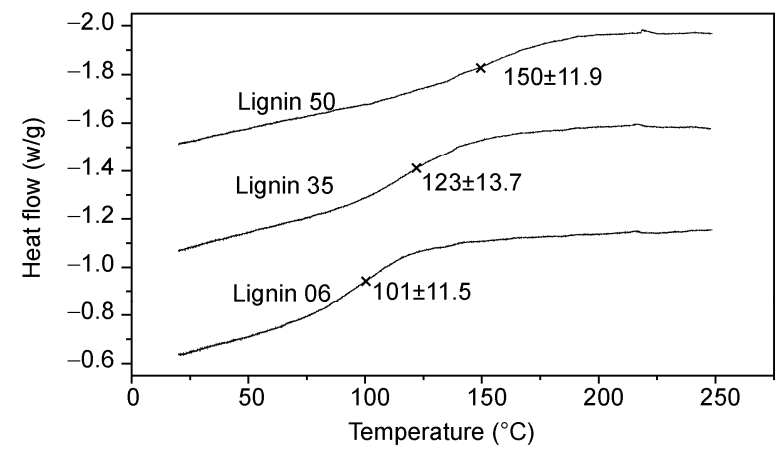

Figure 2 DSC Scans of pyrolytic lignin (heating rate $20^{\circ} \mathrm{C} / \mathrm{min}$ ).

decrease free volume by increasing chain stiffness. The polar groups may also be responsible for the increased thermal stability and ash content. The $T_{\mathrm{g}}$ of pyrolytic lignin is given in Table 4.

To better understand the decomposition of pyrolytic lignin extracted from aged bio-oils under different conditions, the thermal degradation behavior of the pyrolytic lignin was investigated. Typical thermogravimetric (TG) curves of pyrolytic lignin decomposition obtained in this study are shown in Figure 3, where the decomposition begins at around $114-131^{\circ} \mathrm{C}$ and is completed at $591-596^{\circ} \mathrm{C}$. The residues of the different samples are shown in Table 5. The temperature corresponding to $5 \mathrm{wt} \%$ of weight loss and final residue increased with the treatment temperature of bio-oils. The higher WIF and possible larger molecular weight distribution from aging at higher temperature may correspond to a higher initial decomposition temperature and a higher temperature for a given weight loss.

The ${ }^{1} \mathrm{H}$ NMR spectra of the pyrolytic lignin samples are

Table 4 Glass transition temperature of pyrolytic lignin at a heating rate of $20^{\circ} \mathrm{C} / \mathrm{min}$

\begin{tabular}{cc}
\hline Samples & $T_{\mathrm{g}}\left({ }^{\circ} \mathrm{C}\right)$ \\
\hline Lignin 06 & $105 \pm 10$ \\
Lignin 35 & $121 \pm 14$ \\
Lignin 50 & $149 \pm 12$ \\
\hline
\end{tabular}

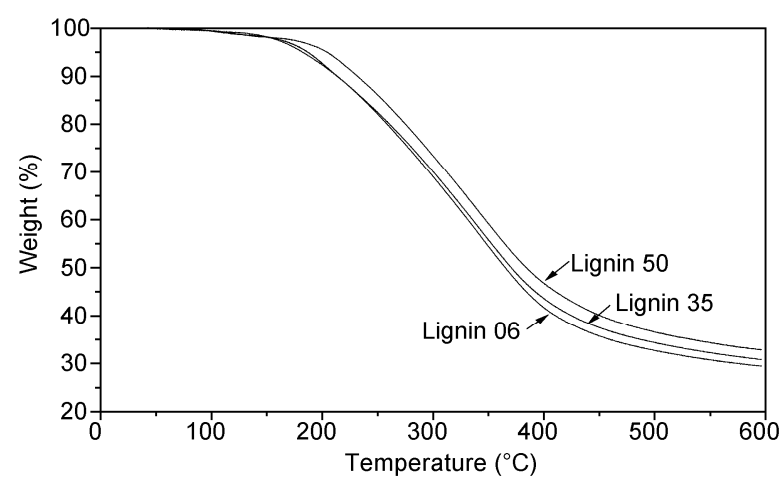

Figure 3 TGA of pyrolytic lignin from bio-oil stored at various temperatures.
Table 5 TGA Results of pyrolytic lignin

\begin{tabular}{ccc}
\hline Samples & Temperature at $95 \mathrm{wt} \%$ weight loss $\left({ }^{\circ} \mathrm{C}\right)$ & Residue $(\mathrm{wt} \%)$ \\
\hline Lignin 06 & $182 \pm 3$ & $30 \pm 1$ \\
Lignin 35 & $191 \pm 5$ & $32 \pm 1$ \\
Lignin 50 & $204 \pm 1$ & $34 \pm 1$ \\
\hline
\end{tabular}

illustrated in Figure 4 and summarized in Table 6. The peaks indicate the proton in the aromatic ring of the Guaiacol-based unit (6.80-7.25 ppm), the protons between 3.48 and $4.00 \mathrm{ppm}$ are most likely due to the presence of a methoxy group, while those between 2.22 and $2.50 \mathrm{ppm}$ are protons in aromatic acetate. The hydrocarbon protons exist between 1.30 and $1.60 \mathrm{ppm}$ and the aliphatic protons in $\mathrm{CH}_{3}, \mathrm{CH}_{2}$ and $\mathrm{CH}$ groups bonded to $\mathrm{C}$ are found from 0.8 to $1.6 \mathrm{ppm}$. Integration results for the hydroxyl peaks from ${ }^{1} \mathrm{H}-\mathrm{NMR}$ spectra of the acetylated WIF (pyrolytic lignin) fractions are listed in Table 6 . There is no significant difference between the samples aged at 6 and $35^{\circ} \mathrm{C}$, but the sample aged at $50^{\circ} \mathrm{C}$ shows decreases in both aliphatic and aromatic hydroxyl groups, relative to methoxyl groups.

There seems to be sufficient evidence to support the conclusion that changes are occurring in the pyrolytic lignin upon aging of the bio-oil. Although physicochemical changes in the bio-oil are undesirable as a fuel, the extraction of pyrolytic lignin for producing value-added chemicals may pose another opportunity. Further study is needed to explore this potential.

\section{Discussion}

Pyrolytic lignin is a homogeneous, water-insoluble powder

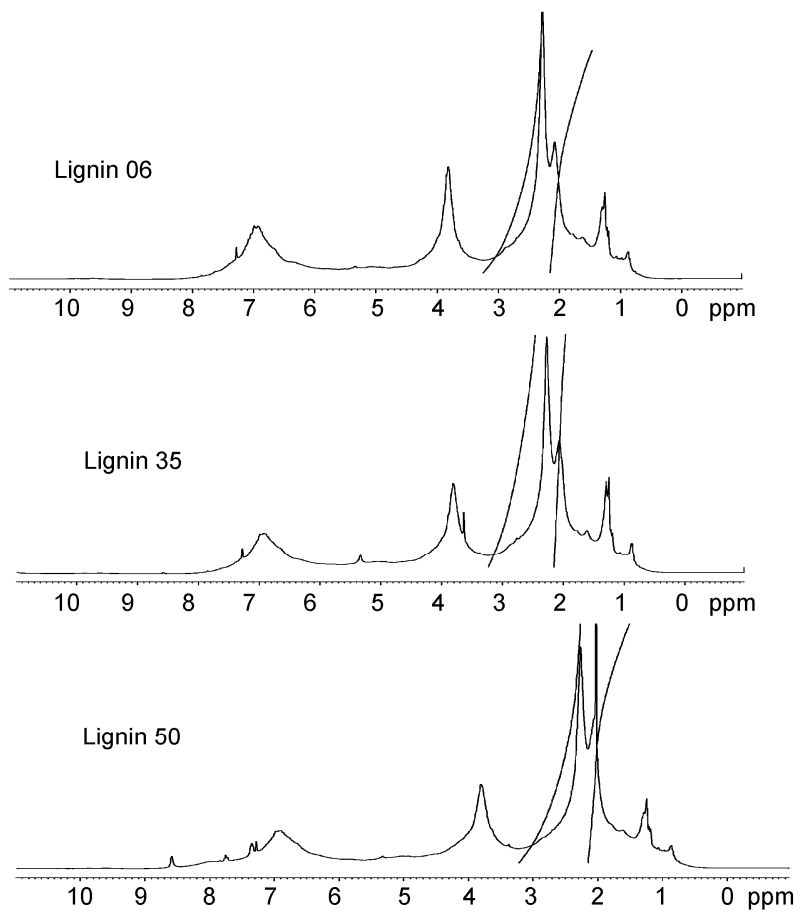

Figure $4 \quad{ }^{1}$ H-NMR of acetylated pyrolytic lignin from bio-oil stored at various temperatures. 
Table $6 \quad{ }^{1} \mathrm{H}$ NMR spectra of pyrolytic lignin

\begin{tabular}{|c|c|c|c|c|c|}
\hline \multirow{2}{*}{ ID } & \multirow{2}{*}{ Range (ppm) } & \multicolumn{3}{|c|}{ Max value of peak (ppm) } & \multirow{2}{*}{ Representation } \\
\hline & & Lignin 06 & Lignin 35 & Lignin 50 & \\
\hline 1 & $7.25-6.80$ & 7.01 & 6.95 & 6.90 & Proton in aromatic ring of Guaiacol-based unit \\
\hline 2 & $4.00-3.48$ & 3.82 & 3.82 & 3.81 & Methoxy group protons \\
\hline 3 & $2.50-2.22$ & 2.30 & 2.30 & 2.28 & Protons in aromatic acetate \\
\hline 4 & $1.60-1.30$ & 1.30 & 1.25 & 1.27 & Hydrocarbon protons \\
\hline 5 & $0.80-1.30$ & 0.88 & 0.85 & 0.86 & Aliphatic protons in $\mathrm{CH}_{3}, \mathrm{CH}_{2}$ and $\mathrm{CH}$ groups \\
\hline
\end{tabular}

extracted from bio-oil. The yield and average molecular weight distribution $(700-1000 \mathrm{~g} / \mathrm{mol})$ of the pyrolytic lignin increases with the aging temperature of bio-oil. These changes are also reflected in an increase in the $T_{\mathrm{g}}$, in the GPC analysis, and initial decomposition temperature upon thermal degradation through TG curves with increasing aging temperature. The FTIR data shows that a lower aging temperature favors hydroxyl formation, through the $\mathrm{O}-\mathrm{H}$ stretching region (3000-3600 $\mathrm{cm}^{-1}$ ). The increasing $\mathrm{C}-\mathrm{O}$ and carbonyl, $\mathrm{C}=\mathrm{O}$, peaks with increasing aging temperature indicate possible oxidation reactions occurring during aging. This is further supported by the change in the oxygen content observed through the elemental analysis. NMR results show that lignin extracted from the bio-oil sample aged at $50^{\circ} \mathrm{C}$ decreased in both aliphatic and aromatic hydroxyl groups relative to methoxyl groups, further confirming the trend seen in FTIR data of the lower temperature being more favorable to hydroxyl formation.

This work was supported by the National Basic Research Program of China (2007CB210208), the National S\&T Major Project of China (2008ZX07101), the China Scholarship Council (CSC), NSERC (the Natural Science and Engineering Research Council of Canada), BIOCAP and CFI (Canadian Funding for Innovations).

1 Bridgwater A V, Peacocke G V C. Fast pyrolysis processes for bio- mass. Renew Sust Energ Rev, 2000, 4: 1-73

2 Mohan D, Pittman C U, Steele P H. Pyrolysis of wood/biomass for bio-oil: A critical review. Energy Fuel, 2006, 20: 848-889

3 Huber G W, Iborra S, Corma A. Synthesis of transportation fuels from biomass: Chemistry, catalysts and engineering. Chem Rev, 2006, 106: 4044-4098

4 Oasmaa A, Kuoppala E, Selin J F, et al. Fast pyrolysis of forestry residue and pine. (4). Improvement of the product quality by solvent addition. Energy Fuel, 2004, 18: 1578-1583

5 Diebold J P, Czernik S. Additives to lower and stabilize the viscosity of pyrolysis oils during storage. Energy Fuel, 1997, 11: 1081-1091

6 Scholze B, Hanser C, Meier D. Characterization of the waterinsoluble fraction from fast pyrolysis liquids (pyrolytic lignin)—Part II: GPC, carbonyl groups, and ${ }^{13} \mathrm{C}-\mathrm{NMR}$. J Anal Appl Pyrolysis, 2001, 58-59: 387-400

7 Scholze B, Meier D. Characterization of the water-insoluble fraction from pyrolysis oil (pyrolytic lignin). Part I: PY-GC/MS, FTIR, and functional groups. J Anal Appl Pyrolysis, 2001, 60: 41-54

8 Scholze B, Meier D. Characterization of the water-insoluble fraction from pyrolysis oil (pyrolytic lignin). Part IV: Structure elucidation of oligomeric molecules. J Anal Appl Pyroly, 2009, 85: 98-107

9 Ba T, Chaala A, Garcia-Perez M, et al. Colloidal properties of bio-oils obtained by vacuum pyrolysis of softwood bark: Aging and thermal stability. Energy Fuel, 2004, 18: 188-201

10 Czernik S, Johnson D K, Black S. Stability of wood fast pyrolysis oil. Biomass Bioenerg, 1994, 7: 187-192

11 Panagiotis N. Binders for the wood industry made with pyrolysis oil. PyNe Newsletter 6, 1998

12 Sipilad K, Kuoppala E, Fagernas L, et al. Characterization of biomass-based flash pyrolysis oils. Biomass Bioenerg, 1998, 14: 103-113

Open Access This article is distributed under the terms of the Creative Commons Attribution License which permits any use, distribution, and reproduction in any medium, provided the original author(s) and source are credited. 